\title{
Sintomas de TEPT e trauma na infância em pacientes com transtorno da personalidade bordeline
}

Symptoms of ptsd and trauma in childhood in patients with borderline personality disorder

Síntomas de tept y trauma en la infancia en pacientes con trastorno límite de la personalidad

\author{
Isadora Klamt da Conceição* \\ Joana Rodrigues Bello* \\ Christian Haag Kristensen ${ }^{* * *}$ \\ Vinícius Guimaräes Dornelles ${ }^{* * *}$
}

\begin{abstract}
Resumo
$\mathrm{O}$ transtorno da personalidade borderline (TPB) é o mais prevalente dentre todos os transtornos da personalidade, apresentando um percentual de 5,9\% na população geral. O transtorno tem um impacto enorme na vida dos sujeitos, por isso a comunidade científica está voltada a estudar elementos relacionados a essa psicopatologia. Tendo isso em vista, os objetivos deste artigo foram: investigar a presença de sintomatologia pós-traumática e o histórico de trauma na infância em sujeitos com TPB. Para isso, foi realizado um estudo transversal, de caráter exploratório. Dessa forma, 22 sujeitos com diagnóstico de TPB preencheram um instrumento de rastreio para avaliar sintomas de TEPT. Foi encontrado que os pacientes com TPB apresentam diversos sintomas de TEPT, como dificuldade de concentração, lembranças intrusivas, tensão ao relembrar e evitação de lembranças.
\end{abstract}

Palavras-chave: Transtorno da personalidade borderline. Transtorno da personalidade borderline e trauma. Transtorno da personalidade borderline e transtorno de estresse pós-traumático.

\footnotetext{
Texto recebido em abril de 2012 e aprovado para publicação em maio de 2014.

"Mestra em Psicologia, área Cognição Humana, pela Pontifícia Universidade Católica do Rio Grande do Sul (PUC RS), especialista em Psicoterapia Cognitivo-comportamental pela WP Centro de Psicoterapia Cognitivo Comportamental, psicóloga. Endereço: Rua Barão do Amazonas, 1049, ap. 204, Porto Alegre-RS. CEP: 90670-004. E-mail: isadoraklamt@gmail.com.

"Formação em Terapia do Esquema pela Wainer Psicologia Cognitiva e NYC Institute of Schema Therapy, treinamento em Psicoterapia Cognitivo-Comportamental nível I pelo Beck Institute, formação em Transtornos Alimentares e Obesidade pela Wainer Psicologia Cognitiva, psicóloga. Endereço: Avenida Borges de Medeiros, 2500, sala 911 - Praia de Belas, Porto AlegreRS. CEP: 90110-150. E-mail: joana.r.bello@gmail.com.

*** Doutor em Psicologia do Desenvolvimento pela Universidade Federal do Rio Grande do Sul (UFRGS), com estágio no exterior na University of Arizona (USA), mestrado em Psicologia do Desenvolvimento pela UFRGS, especialista em Neuropsicologia (CRP/07), formado em Terapia Cognitiva pelo Beck Institute for Cognitive Therapy and Research (USA), psicólogo. Bolsista de Produtividade em Pesquisa do CNPq - Nível II. Endereço: Programa de Pós-Graduação em Psicologia da Pontifícia Universidade Católica do Rio Grande do Sul. Avenida Ipiranga, 6681, prédio 11, Porto Alegre-RS. CEP: 90619-900. E-mail: christian.kristensen@pucrs.br.

**** Mestre em Psicologia, área de concentração de Cognição Humana (PUC RS), formado em Terapias Baseadas em Evidências para o Transtorno da Personalidade Borderline (FORO/ARG), especialista em Terapias Cognitivo-Comportamentais, coordenador do GEP, graduado no Dialectical Behavior Therapy - Intensive Training (Behavioral Tech/EUA e Foro/ARG), professor da Faculdade de Psicologia da Unisinos, coordenador do Curso de Formação em Terapias Comportamentais Contextuais do InTCC, professor e supervisor do curso de Especialização em Terapias Cognitivo-comportamentais do InTCC, sócio do InTCC e sócio da International Society for the Study of Personality Disorders, psicólogo. Endereço: Rua Jari, 740, ap. 808, torre A - Passo D’Areia, Porto Alegre-RS. CEP: 91350-170. E-mail: vinicius.dornelles@gmail.com.
} 


\begin{abstract}
The borderline personality disorder (BPD) is the most prevalent of all diagnostic personality disorders, presenting a percentage of $5.9 \%$ in the general population. The disorder has a huge impact in the life of the individuals, for this reason the scientific community is studying the relation elements of this psychopathology. The study's purpose was to investigate the presence of posttraumatic symptomatology and history of childhood trauma. The work's delineation was a transversal study with exploratory character. For these 22 borderline patients completed a screening task in order to evaluate symptoms of PTSD. It was found that the borderline patients presented several symptoms of PTSD, such as: difficulty in concentration, intrusive memories, tension in remembering and avoidance of memories.
\end{abstract}

Keywords: Borderline personality disorder. Borderline personality disorder and trauma. Borderline personality disorder and posttraumatic stress disorder (PTSD).

\title{
Resumen
}

El trastorno límite de la personalidad (TLP) es el más frecuente entre todos los trastornos de la personalidad, presentando un porcentaje de 5,9\% en la población general. El trastorno tiene un gran impacto en la vida de los individuos, es por esto que la comunidad científica está dedicada a estudiar elementos relacionados con esta psicopatología. Considerando esto, los objetivos del presente artículo fueron: investigar la presencia de sintomatología post-traumática y el historial de trauma en la infancia de personas con TLP. Para esto, se realizó un estudio transversal con carácter exploratorio. De esa manera, 22 personas con TLP respondieron un formulario de acompañamiento para evaluar los síntomas de TEPT. Se encontró que los pacientes con TLP tienen muchos síntomas de TEPT, tales como: dificultad para concentrarse, recuerdos intrusivos, tensión al recordar y evitar recuerdos.

Palabras clave: Trastorno límite de la personalidad. Trastorno límite de la personalidad y trauma. Trastorno límite de la personalidad y trastorno de estrés postraumático. 


\section{Introdução}

0 termo borderline foi utilizado pela primeira vez em 1884, por Hughes, para diferenciar pessoas que passavam a vida entre a linha tênue da sanidade e da loucura (Helgeland \& Torgersen, 2004). Conceitualmente, borderline foi descrito por Stern, em 1938, e por Knight, em 1940. Posteriormente, a etiologia borderline foi estudada por Kernberg e Masterson, na década de 1960, e por estudos em fenomenologia por Grinker e Gunderson, em 1979. Inicialmente a patologia Borderline era classificada como esquizofrenia, e, posteriormente, a tentativa foi de pensá-la como um transtorno atípico de humor (Kaplan \& Sadock, 1999; Linehan, 2010).

Kernberg, Selzer, Koenigsberg, Carr e Appelbaum (1991) sugeriram uma organização borderline de personalidade com três características: difusão de identidade (falta de integração do conceito de self e outros significativos), nível de operações defensivas (clivagem e outros mecanismos) e incapacidade no teste realidade. Assim, nessa perspectiva, a organização borderline estaria associada aos conceitos de separação e individuação. Como visto, essa descrição ainda era bastante abrangente, pouco específica e incluía outros transtornos mentais. Entretanto somente a partir da terceira edição do Manual Diagnóstico e Estatístico de Transtornos Mentais (American Psychiatric Association [APA], 1987) que o transtorno da personalidade borderline (TPB) foi formalmente reconhecido como uma categoria nosológica específica. Na revisão de texto da quarta edição do DSM (American Psychiatric Association [APA], 2002), a base do conceito foi mantida, tendo como foco a fragilidade nos relacionamentos interpessoais, autoimagem prejudicada, instabilidade de humor e acentuada impulsividade (Beck, Freeman \& Davis, 2005). A definição hoje utilizada no DSM-IV-TR (2002) é de que os sujeitos com TPB apresentam um padrão global de instabilidades nos relacionamentos interpessoais, da autoimagem e dos afetos e acentuada impulsividade, que se manifesta no início da idade adulta e está presente em uma variedade de contextos. Para o diagnóstico ser realizado, os sujeitos devem apresentar, no mínimo, cinco dos seguintes critérios:

a) esforços frenéticos no sentido de evitar um abandono real ou imaginário;

b) um padrão de relacionamentos interpessoais instáveis e intensos, caracterizado pela alternância entre extremos de idealização e desvalorização;

c) perturbação da identidade: instabilidade acentuada e resistente da autoimagem ou do sentimento de self; 
d) impulsividade em pelo menos duas áreas potencialmente prejudiciais à própria pessoa;

e) recorrência de comportamento, gestos ou ameaças suicidas ou de comportamento automutilante;

f) instabilidade afetiva devido a uma acentuada reatividade do humor;

g) sentimentos crônicos de vazio;

h) raiva inadequada e intensa ou dificuldade em controlar a raiva; e

i) ideação paranoide transitória ao estresse ou graves sintomas dissociativos (American Psychiatric Association, 2002).

Cabe salientar que a apresentação do diagnóstico de TPB, assim como dos demais transtornos da personalidade (TP) no DSM-V, está colocada em duas seções distintas do manual, a seção II e a seção III. Na seção II, a qual corresponde à versão oficial do manual, os critérios diagnósticos previstos para o TPB permanecem exatamente os mesmos do DSM-IV-TR. Contudo a seção III aponta uma proposta de revisão baseada em uma avaliação dimensional dos TP e não mais categórica. Nessa abordagem, ainda em estudo pelo grupo de trabalho dos TP do DSM-V, existiriam cinco domínios gerais de traços da personalidade: afetividade negativa (vs. estabilidade emocional), distanciamento (vs. extroversão), antagonismo (vs. agradabilidade), desinibição (vs. conscienciosidade) e psicotismo (vs. lucidez). Esses cinco domínios foram inspirados na teoria dos cinco grandes fatores da personalidade, cuja validade é demonstrada por uma ampla gama de estudos. Dentro desses cinco domínios gerais estariam agrupados 25 traços específicos: afetividade negativa (labilidade emocional, ansiosidade, insegurança de separação, submissão, hostilidade, perseveração, depressividade, desconfiança e afetividade restrita); distanciamento (isolamento, evitação de intimidade, anedonia, depressividade, afetividade restrita e desconfiança); antagonismo (manipulação, enganador, grandiosidade, busca de atenção e insensibilidade); desinibição (irresponsabilidade, impulsividade, distratabilidade, assumir riscos e falta de perfeccionismo rígido); psicotismo (pensamentos e experiências não habituais, excentricidade e desregulação cognitiva e perceptual). A avaliação de um dado TP, de acordo com esse sistema, seria realizada mediante uma escala denominada Escala do Nivel de Funcionamento da Personalidade (LPFS - Level of Personality Functioning Scale). Essa diferenciaria cinco níveis: $0=$ pouco ou nenhum comprometimento; 1 = pouco comprometimento; 2 = moderado comprometimento; 3 = severo comprometimento; e 4 = comprometimento 
extremo. Assim, o diagnóstico de um dado TP ocorreria a partir do nível 2 da escala. Dessa forma, os critérios diagnósticos para TPB, dentro dessa sistemática, ficariam agrupados da seguinte maneira: (A) Moderado ou grave comprometimento do funcionamento da personalidade caracterizado pela dificuldade em duas ou mais das quatro áreas seguintes: (1) identidade - autoimagem marcadamente pobre, pouco desenvolvida ou instável, por vezes associada à autocrítica exagerada, sentimentos crônicos de vazio e sintomas dissociativos decorrentes de estresse; (2) auto-orientação - instabilidade em objetivos, aspiraçōes, valores ou planos de carreira; (3) empatia - déficits na habilidade de reconhecer as emoçõos e necessidades dos outros, associada à hipersensibilidade interpessoal, e percepção seletiva dos outros em termos de atributos negativos ou vulnerabilidades; (4) intimidade - relações próximas intensas, instáveis e conflituosas, marcadas por desconfiança, carência e preocupações ansiosas com abandonos reais ou imaginários. Os relacionamentos próximos tendem a ser vistos em extremos de idealização e desvalorização, e alternando entre envolvimento intenso e distanciamento. (B) quatro ou mais dos sete traços de personalidade patológicos, sendo que pelo menos um deles deve ser (5) impulsividade, (6) assumir riscos ou (7) hostilidade: (1) labilidade emocional; (2) ansiosidade; (3) insegurança de separação; (4) depressividade (esses quatro traços são pertencentes ao domínio de afetividade negativa); (5) impulsividade; (6) assumir riscos (esses dois traços são pertencentes ao domínio de desinibição); (7) hostilidade (pertencente ao domínio antagonismo) (American Psychiatric Association [APA], 2013; Esbec \& Echeburúa, 2011).

No que se refere ao impacto pessoal do TPB, sabe-se que é um transtorno que traz muito sofrimento para as pessoas. Cerca de 8 a 10\% dos sujeitos com TPB cometem suicídio, além de terem severos prejuízos ocupacionais e sociais devido ao transtorno. Estima-se $10 \%$ das pessoas que buscam atendimento em saúde mental apresentam o transtorno (APA, 2002).

O curso do TPB apresenta certa variabilidade, sendo mais comum uma instabilidade crônica no início da adultez, com episódios de descontrole afetivo e impulsivo, com uma tendência a adquirir alguma estabilidade na faixa dos 30 e 40 anos (APA, 2002). Os tratamentos com maior apoio empírico para o TPB são a terapia comportamental dialética (Linehan, 2010) e a terapia do esquema (Young, Klosko \& Weishaar, 2008).

A etiologia do TPB é comprovadamente multifatorial, havendo interação entre fatores genéticos e ambientais. Estudos psicobiológicos com esses pacientes indicam que pode haver uma desregulação no sistema serotoninérgico (Linehan, 2010). Já em uma perspectiva psicodinâmica, o TPB parece estar relacionado à dissociação ou cisão da agressividade, as quais teriam origem em crianças 
excessivamente agressivas ou pais muito frustrantes. Outro fator relevante parece ser a incapacidade dos pais em prover cuidados necessários e validar adequadamente os sentimentos de suas crianças (Kernberg \& Michels, 2009; Beck, Freeman \& Davis, 2005; Scott, Levy \& Pincus, 2009). Além dessas, também se consideram alterações encefálicas funcionais e estruturais (Oldaham, 2009).

Do ponto de vista ambiental, diversos estudos associam traumas na infância (tais como abuso físico e, ou, sexual) com ocorrência de TPB (Florenzano et al., 2002; Gunderson, 2009; Sansone \& Sansone, 2009; Ball \& Links, 2009; Pagano et al., 2004; Johnson, Gentile \& Correll, 2010). O estudo realizado por Florenzano et al. (2002) mostrou que $63,1 \%$ das pacientes referiram ter apresentado pelo menos um acontecimento traumático na infância, 22,9\% recordaram mais de um acontecimento traumático e 16,4\%, mais de quatro acontecimentos traumáticos durante a infância. Por sua vez, Johnson, Gentile e Correl (2010) também confirmam níveis especialmente altos de trauma infantil. Em relação aos pacientes com TBP, $71,4 \%$ recordaram trauma infantil de algum tipo, em comparação com $28,6 \%$ que não recordaram. A estimativa de Fruzzetti, Shenk \& Hoffman (2005) é ainda menos conservadora. Segundo os autores, a ocorrência de abuso sexual é de $75 \%$ nos pacientes com diagnóstico de TPB. Entretanto histórico de abuso e, ou, negligência não é uma característica exclusiva de TPB, mas de todos os transtornos da personalidade de cluster $\mathrm{B}$ (Johnson, Smailes, Philip \& Cohen, 2000).

O TPB apresenta altos índices de comorbidade com outros transtornos da personalidade, tais quais transtorno da personalidade esquizotípica $(14,3 \%)$, transtorno da personalidade antissocial $(8,3 \%)$ e transtorno da personalidade paranoide $(14,5 \%)$ (Watson \& Sinha, 1998). Outra coocorrência se deu com transtorno da personalidade esquiva (59\%) e transtorno da personalidade dependente (45\%) (Zanarini, Frankenburg \& Vujanovic, 2004). Em um estudo de Helgeland e Torgersen (2004), 36\% dos casos dos pacientes borderline tinham comorbidade com transtorno da personalidade antissocial e $28 \%$ tinham comorbidade com transtorno da personalidade depressiva.

Com frequência se pode realizar o diagnóstico de TPB conjuntamente com outros transtornos de Eixo I (48\% a 54\% dos pacientes), como transtorno do pânico, fobia social e transtorno do estresse pós-traumático. Em relação a demais transtornos específicos, $61 \%$ tinham transtorno depressivo maior; $41 \%$, distimia; 29\%, transtorno do pânico (Zanarini, Gunderson, Frankenburg \& Chauncey, 1989; Zlotnick, Franklin \& Zimmerman, 2002; Gunderson, 2009); e 28\% , transtorno de alimentação sem outra especificação. 
De especial interesse para este estudo está a comorbidade entre TPB e TEPT. Considerando a possível combinação de trauma na etiologia do TPB, não surpreende que essa comorbidade seja elevada. Os eventos traumáticos na infância, como o incesto ou outras formas de maus-tratos, podem predispor o indivíduo à manifestação de TEPT com TPB (Kudler, 1993). De fato, estudos empíricos estimam comorbidade de 56\% a 68\% entre TPB e TEPT (Zlotnick, Franklin \& Zimmerman, 2002).

Além disso, nesse mesmo estudo, foi mostrado que os dois distúrbios compartilham de histórico de abuso sexual na infância (Zanarini, 1998, apud Hodges, 2003). Já Golier et al. (2003) mostraram que indivíduos com TPB apresentaram uma tendência maior a ter TEPT do que os participantes sem o transtorno, porém a amostra também apontou que altos níveis de TEPT foram encontrados em indivíduos com transtorno da personalidade paranoide, sugerindo não haver uma relação única entre TPB e TEPT.

Quanto aos sintomas que ambas as enfermidades têm em comum (Herman \& Van der Kolk, 1987, apud Woodward, Taft, Gordon \& Meis, 2009), consideramse a agressividade para si e para com os outros, o fato de serem irritadiços, incapazes de tolerar extremos emocionais disfóricos, ter uma sensação de vazio ou de morte e serem altamente reativos ao estresse leve. Quanto às diferenças nos sintomas, os pacientes com TEPT são mais prováveis de estarem relacionados a respostas situacionais em relação a circunstâncias externas (Becker, 1999, apud Woodward et al., 2009). Além disso, a American Psychiatric Associtation (2002) mostra que sintomas como flashbacks, evitação, resposta exagerada a algum estímulo que lembre a situação traumática não são critérios do TPB. Já pacientes com TPB podem apresentar distúrbios de identificação e maus-tratos consigo próprios, os quais não fazem parte dos critérios de TEPT (Woodward et al., 2009).

Conforme Hodges (como citado em Pagura et al., 2010) apesar de comórbidos, TEPT e TPB não são totalmente sobrepostos. Ainda que apresentem similaridades em alguns aspectos, esses transtornos se diferenciam nos sintomas, funcionamento, resposta terapêutica, etc. Alguns autores defendem que o TPB é, na verdade, uma forma crônica de TEPT que se tornou integrada na personalidade da pessoa (Landecker, 1992; Zimmerman \& Matia, 1999, apud Hodges, 2003). Entretanto, Hodges (2003) pontua que nem todas as mulheres diagnosticadas com TPB foram sexualmente ou fisicamente traumatizadas e que múltiplos fatores podem produzir a sintomatologia borderline. Do ponto de vista clínico, observa-se que sujeitos com TPB e TEPT comórbidos apresentam um quadro mais grave do que aqueles com somente um dos transtornos (Zlotnick, Franklin \& Zimmerman, 2002). 
Outra questão importante é que diversas pesquisas demonstram que o diagnóstico de TEPT muitas vezes é realizado para não colocar no paciente o mau rótulo e o peso associado ao TPB (Hodges, 2003; Gunderson, apud Woodward et al., 2009).

Considerando (a) a ocorrência de trauma como uma condição necessária para o diagnóstico de TEPT e provável fator etiológico para TPB e (b) a elevada comorbidade entre esses transtornos, este estudo exploratório objetivou investigar história de trauma infantil e sintomas de TEPT em pacientes com TPB.

\section{Método}

\section{Delineamento}

Foi realizado um estudo transversal com 22 pacientes portadores de TPB. Os participantes foram recrutados por conveniência, indicados por profissionais da área e que trabalham em instituições de saúde mental.

\section{Procedimento de coleta}

Após a aprovação pelo Comitê de Ética Científico da Pontifícia Universidade Católica do Rio Grande do Sul, entrou-se em contato com as instituições de saúde mental da cidade de Porto Alegre-RS. Conversou-se com psicólogos e psiquiatras destas para o início do recrutamento dos participantes. Posteriormente, realizouse contato pessoal do pesquisador com os pacientes, solicitando a participação na pesquisa mediante a explicação da proposta para estes. Então os pacientes que concordaram em participar receberam o termo de consentimento livre e esclarecido, no qual foram explicados os procedimentos éticos. Realizado esse primeiro contato, foi iniciada a aplicação dos instrumentos.

Para ser inclusos no estudo, os sujeitos deveriam apresentar diagnóstico de TPB apontado pelos clínicos e confirmado pela aplicação da DIB-R (entrevista para diagnóstico de transtorno da personalidade borderline - revista; Zanarini et al., 1989), ter idade entre 18 e 50 anos, não apresentar retardo mental, esquizofrenia, depressão psicótica, transtorno do humor bipolar (tipo 1), abuso de drogas nos últimos seis meses e risco de suicídio. Pacientes com escores $\geq$ 36 pontos no $\mathrm{BDI}$, indicando depressão grave, foram excluídos assim como pacientes com escores $\geq 2$ pontos na questão 9 ("ideação suicida") no mesmo teste. Da mesma maneira, foram excluídos do estudo pacientes com escores $\geq$ 31 pontos no inventário Beck de ansiedade (BAI), indicando grave nível de ansiedade. A avaliação dos critérios de exclusão da amostra foi feita de duas formas: 
a) dados do prontuário do paciente; e

b) o instrumento DIB-R avalia a possível existência de transtorno do humor bipolar tipo 1, sendo que, caso o paciente apresente essa patologia, o instrumento não permite que se faça o diagnóstico de TPB; assim, ele estaria naturalmente excluído do estudo. Da mesma forma, a DIB-R avalia sintomas psicóticos condizentes com o quadro de esquizofrenia, assim como outros transtornos psicóticos. Da mesma forma, uma vez que esses critérios sejam preenchidos, o instrumento não permite $o$ diagnóstico de TPB, assim esse paciente estaria excluído da amostra desse estudo.

\section{Instrumentos}

Foram utilizados os instrumentos DIB-R (entrevista para diagnóstico de transtorno da personalidade borderline - revista) (Zanarini et al., 1989). O DIB-R se trata de uma entrevista semiestruturada com quatro fatores distintos:

a) seção afeto - está relacionado à maneira da pessoa entender seus sentimentos e seu humor;

b) seção cognição - para saber se há ou não perturbações do pensamento, tais como delírios e alucinações;

c) seção padrão de ações impulsivas - para verificar se ocorrem comportamentos impensados e, ou, autodestrutivos; e

d) seção relacionamentos interpessoais:

$\checkmark$ no qual se analisa a possível instabilidade dos relacionamentos com os outros bem como sua dificuldade de manter apego saudável. Cada fator tem questôes que variam entre " 0 - não apresenta o sintoma, 1 - provável que apresente o sintoma e 2 - apresenta o sintoma”. Todos os fatores têm um determinado número de questóes que tem de ser pontuadas com os números 1 ou 2 para que a seção seja pontuada (escore graduado). Assim, para fechar o diagnóstico de $\mathrm{TPB}$, é necessário que se pontue pelo menos três dos quatro fatores, somando, no total, 8 ou mais pontos no score graduado. 
Childhood trauma questionnaire (CTQ, em português - Questionário sobre traumas na infância - Quesi, traduzido para o português por Grassi-Oliveira, Stein \& Pezzi, 2006). É uma escala de autorrelato que avalia a presença de histórico de situações de abuso ou negligência durante a infância do indivíduo. Essa escala está dividida em cinco subgrupos, sendo: abuso emocional, abuso físico, abuso sexual, negligência física e negligência emocional.

Screening for posttraumatic stress symptoms (SPTSS; Carlson, 2001; versão em português por Kristensen, 2005). O SPTSS é uma medida de rastreio breve e de autorrelato, composta por 17 itens, apresentados na primeira pessoa do singular, para avaliar a presença de sintomas de TEPT. Nesse instrumento, avaliam-se os itens de acordo com três fatores: evitação, revivência e excitabilidade.

\section{Participantes}

Participaram deste estudo 22 pacientes de ambos os sexos (85,7\% mulheres e $14,3 \%$ homens), com idade entre 18 e 50 anos $(M=33,33 ; D P=10,781)$. Os participantes foram recrutados por conveniência, indicados por profissionais da área (psicólogos e psiquiatras) que trabalham em instituiçôes de saúde mental. Quanto ao grau de escolaridade dos participantes, 2 sujeitos (9,5\%) apresentam ensino fundamental incompleto; $1(4,8 \%)$, ensino médio incompleto; 6 $(28,6 \%)$, ensino médio completo; $6(28,6 \%)$, ensino superior incompleto; 2 $(9,5 \%)$, ensino superior completo; e 4 (19\%), pós-graduação completa.

\section{Critérios de análise dos dados}

Os dados foram analisados por meio de estatística descritiva, indicando as pontuações, média, desvio padrão e mínima/máxima dos itens importantes para os objetivos do trabalho. Depois se correlacionaram as escalas analisadas bem como suas sub-seções. As correlações foram feitas utilizando-se o programa SPSS versão 17. A análise correlacional foi feita conforme Spearman (correlação de espécie sig 1-tailed - relação monocaudal) entre os testes DIB-, Quesi e SPTSS. Essa análise faz uma correlação entre as variáveis, de modo que não se trata de uma relação causal entre estes, entretanto demonstra as coocorrências entre os instrumentos. Dessa forma, quando se percebe uma correlação direta entre os instrumentos, a amostra expressa que o aumento em uma variável correlacionada indica valor aumentado também na outra variável. 


\section{Resultados}

Em relação à sintomatologia borderline, verificou-se que os pacientes avaliados apresentaram grande dificuldade de relacionamento interpessoal (ver tabela 1), assim como em afetividade e cognição. Porém o grau de intensidade desses fatores foi menor se comparados aos padrões de relacionamentos interpessoais. Já o fator padrões de impulsividade foi o menos pontuado.

Tabela 1. Descrição das variáveis de interesse

\begin{tabular}{|c|c|c|c|c|c|}
\hline Variável & Média & $\begin{array}{l}\text { Desvio } \\
\text { Padrão }\end{array}$ & $\begin{array}{c}\text { Dados } \\
\text { Normativos }\end{array}$ & Mínima & Máxima \\
\hline Tdade (anos) & 33.33 & 10.78 & -- & & \\
\hline Estudo (anos) & 14.74 & 4.04 & -- & 8,5 & 26,5 \\
\hline DIB-R & & & & & \\
\hline Afetividade & 42.80 & 6.40 & & & \\
\hline Cognição & 40.47 & 5.45 & & & \\
\hline $\begin{array}{l}\text { Padrões de. } \\
\text { Impulsividade }\end{array}$ & 28.42 & 4.38 & & & \\
\hline $\begin{array}{l}\text { Relacionamento. } \\
\text { Interpessoal }\end{array}$ & 64.61 & 6.53 & & & \\
\hline Score (1-10) & $9,00^{a}$ & $8,50-9,50 b$ & $>8^{\mathrm{a}}$ & 8,00 & 10,00 \\
\hline CTQ & & & & & \\
\hline Total & 51,47 & 17,67 & & 26,00 & 90,00 \\
\hline $\begin{array}{c}\text { Abuso Emocional } \\
\text { Abuso Sexual } \\
\text { Abuso Físico }\end{array}$ & $\begin{array}{l}13.61 \\
6.00 \\
8,00^{\mathrm{a}}\end{array}$ & $\begin{array}{c}5,74 \\
3.52 \\
5,50-11,50 b\end{array}$ & $\begin{array}{l}8.39^{b} \\
5.92^{b} \\
6.39^{b}\end{array}$ & $\begin{array}{l}5,00 \\
5,00 \\
5,00\end{array}$ & $\begin{array}{l}24,00 \\
21,00 \\
18,00\end{array}$ \\
\hline $\begin{array}{l}\text { Negligência } \\
\text { Emocional }\end{array}$ & $17,00^{\mathrm{a}}$ & $8,00-18,00 b$ & $9.22^{b}$ & 5,00 & 22,00 \\
\hline $\begin{array}{l}\text { Negligência Física } \\
\text { SPTSS }\end{array}$ & $9,00^{\mathrm{a}}$ & $5,00-11,50 b$ & $7.00^{\mathrm{b}}$ & 5,00 & 17,00 \\
\hline Fator 1 - Revivência & 4.94 & 2.81 & & 0,00 & 10,00 \\
\hline Fator 2 - Evitação & 4.72 & 3.07 & & 0,14 & 10,00 \\
\hline $\begin{array}{c}\text { Fator } 3 \text { - } \\
\text { Excitabilidade }\end{array}$ & 4.47 & 2.57 & & 0,00 & 9,60 \\
\hline Total & 4,71 & 2,61 & $\geq 5$ & 0,06 & 9,88 \\
\hline
\end{tabular}

Nota: Escores de tendência central e dispersão serão expressos como média e desvio padrão, a não ser em ${ }^{a}=$ Mediana $\mathrm{e}^{\mathrm{b}}=$ intervalo interquartil.

Fonte: elaborado pelos autores. 
Os dados do instrumento Quesi foram examinados para que se possam ressaltar os tipos e as frequências de traumas infantis desses pacientes. Analisando os dados da amostra do CTQ (Quesi - Questionário sobre traumas na infância) percebe-se que ocorreram todos os tipos de abuso nos pacientes analisados. Destaca-se, então, a frequência do abuso emocional e da negligência emocional (ver Tabela 1). Isso demonstra, então, um grau de moderado a severo nesses dois tipos de abuso. Já quanto às subescalas abuso físico, abuso sexual e negligência física, os participantes apresentaram índices de leve a moderado de experiências potencialmente traumáticas. Desse modo, observa-se que $81 \%$ dos pacientes apresentam algum tipo de abuso emocional. A amostra também ressalta que $66,6 \%$ dos pacientes sofreram abuso físico, assim como alto índice de negligência emocional $(71,4 \%)$. No que se refere à negligência física, identifica-se uma porcentagem de $61,9 \%$ nos pacientes entrevistados. Quanto ao abuso sexual, $14,4 \%$ das pessoas da amostra relataram tê-lo sofrido.

Em termos de sintomatologia pós-traumática (SPTSS), nota-se que 47,6\% dos pacientes apresentam sintomas para TEPT, reafirmando, assim, que ambas as enfermidades apresentam sintomas em comum (Herman \& Kolk, 1987, apud Woodward et al., 2009). Percebe-se na amostra (ver Tabela 1) que 57,1\% dos pacientes apresentaram pontuação maior ou igual a 5 no fator (2), indicando, então, tendência a evitar pensamentos e comportamentos relacionados a vivências traumáticas. Outro agrupamento que demonstrou maior gravidade foi o fator (1) de revivência, com $52,4 \%$ dos pacientes referindo os sintomas dessa seção, evidenciando que mais da metade dos pacientes tendem a reviver suas experiências traumáticas. No fator (3), 38,1\% dos pacientes pontuaram acima de 5 nesse agrupamento, demonstrando que um pouco mais de um terço dos pacientes apresentam excitabilidade aumentada. No que se refere aos sintomas, os que mais estão em evidência são: item 6 - dificuldade de concentração, com média de 7,47 $(D P=3,68)$; item 11 - lembranças intrusivas, média de 6,61 $(D P=3,69)$; item 14 - tensão ao relembrar, média de 6,14 $(D P=3,55)$; item 4 - evitação de lembranças, média de $6,00(D P=3,81)$; item 16 - sintomas de estresse a partir de lembranças negativas, média de 5,80 $(D P=4,01)$.

Quanto às correlações com as subescalas do CTQ, percebe-se também correlação direta com negligência física e negligência emocional. Há correlação com abuso emocional como também correlação com a subescala de abuso sexual e o total dos scores graduados da DIB-R. Dessa maneira, pacientes que obtiveram um escore graduado elevado provavelmente também sofreram de abuso sexual. Contudo deve salientar-se aqui que a força da correlação foi baixa $(0,374)$, o que nos leva a uma consideração cautelosa desse resultado. 
Percebe-se que há correlação muito significativa entre negligência física e emocional, bem como negligência emocional e abuso emocional, também há ocorrência significativa entre negligência física e abuso sexual. Observando os dados da amostra, também se ressalta correlação direta entre abuso emocional e abuso físico $(r s=, 434 ; p=0,025)$. Há igualmente uma correlação direta com abuso emocional e abuso sexual $(r s=, 427 ; p=0,027)$, demonstrando que pacientes tanto sofreram abuso emocional como sexual. Quanto ao SPTSS com a DIB-R, percebem-se, conforme o previsto, algumas correlações significativas. Entretanto podemos observar uma correlação negativa com o fator 2 evitação do SPTSS e a soma total dos escores graduados da DIB-R ( $r s=388 ; \mathrm{p}=0,041)$. Por conseguinte, ao fazer uma correlação entre os dados que os pacientes borderline demarcaram no SPTSS e no CTQ, nota-se que não existe uma correlação relevante entre negligência emocional (CTQ) e fator 3 excitabilidade (SPTSS) $(, 385 ; \mathrm{p}=0,042)$. Entretanto é importantíssimo levar esses dados em consideração, uma vez que esse índice pode ser influenciado pelo tamanho da amostra e que ele é de grande relevância para a compreensão dos mecanismos patológicos do TPB.

\section{Discussão}

Os resultados estudados da DIB-R (ver tabela 1) demonstram a grande dificuldade dos pacientes borderline em seus relacionamentos interpessoais, o que enfoca o segundo critério para o diagnóstico de TPB, conforme o DSM IVTR (APA, 2002), o qual pontua um padrão de relacionamentos interpessoais instáveis. Porém não corrobora com grande significância o critério quatro do DSM IV-TR, de "impulsividade em pelo menos duas áreas potencialmente prejudiciais à própria pessoa”, já que a média da amostra foi relativamente baixa se comparada às outras subescalas. Essa baixa pontuação ocorreu, possivelmente, porque os pacientes se encontravam medicados e em tratamento psicoterápico. Já no que se refere à correlação dos dados da escala Quesi (CTQ) com a DIB-R, há correlação direta com CTQ total e a soma dos pontos da DIB-R. Isso demonstra então uma coocorrência de TPB com traumas na infância (ver tabela 2). 
Tabela 2. Correlações entre sintomatologia Borderline, pós-traumática e história de trauma na infância

\begin{tabular}{|c|c|c|c|c|c|c|c|c|}
\hline & 1 & 3 & 4 & 5 & 6 & 7 & 8 & 9 \\
\hline 1 DIB-R total escores & 174 & 092 &,- 034 & ,057, &, $374^{*}$ &,- 325 &,$- 388^{*}$ &,- 063 \\
\hline $\begin{array}{l}2 \text { CTQ - Negligência } \\
\text { física }\end{array}$ & &, $533^{* *}$ &, $646^{* *}$ & ,644* &, $380^{*}$ & ,182 & ,230 & ,314 \\
\hline 3 CTQ - Abuso Físico & & & ,248 &, $434^{*}$ &, 429 & ,258 & , 160 & ,186 \\
\hline $\begin{array}{c}4 \text { CTQ - Negligência } \\
\text { Emocional }\end{array}$ & & & & ,790** & ,225 &, 218 &, 206 &, 353 \\
\hline 5 CTQ - Abuso Emocional & & & & & ,542* & ,174 & ,158 & ,173 \\
\hline 6 CTQ - Abuso Sexual & & & & & &,- 054 &,- 121 &,- 062 \\
\hline 7 Fator 1 - Revivência & & & & & & &, $788^{* *}$ & ,690* \\
\hline $\begin{array}{l}8 \text { Fator } 2 \text { SPTSS - } \\
\text { Evitação }\end{array}$ & & & & & & & & ,741* \\
\hline $\begin{array}{l}9 \text { Fator } 3 \text { SPTSS - } \\
\text { Excitabilidade }\end{array}$ & & & & & & & & \\
\hline
\end{tabular}

Nota: ${ }^{*} p \leq 0,05 ;{ }^{* *} p \leq 0,01$. Coeficiente de correlação por postos de Spearman. DIB-R = Entrevista para Diagnóstico de Transtorno da Personalidade Borderline. CTQ = Childhood Trauma Questionnaire (QUESI); SPTSS = Screening for Posttraumatic Stress Symptoms.

Fonte: elaborado pelos autores.

No que se refere ao histórico de trauma infantil nos pacientes da amostra, pode-se perceber que $81 \%$ dos pacientes sofreram abuso emocional e $71,4 \%$ dos pacientes relataram negligência emocional. Ambos os tipos de trauma estão de acordo com as teorias existentes sobre a etiologia do TPB; também confirmando a hipótese de que crianças que sofreram abuso ou negligência em sua infância têm maior probabilidade de desenvolverem TPB ao longo da vida (Widom, Czaja \& Paris, 2009). Além disso, McLean \& Gallop (2003) demonstraram, em seus estudos, a alta incidência de abuso sexual na infância em pacientes com TPB e uma suposta relação entre crianças que sofreram abuso sexual cedo em suas vidas e o diagnóstico de TPB e também de TEPT. Verifica-se no atual estudo que 14,3\% dos pacientes sofreram abuso sexual na infância. Esse achado também corrobora teorias que reportam que o risco de se desenvolver TPB é 14 vezes mais alto em crianças que sofreram abuso sexual (Cohen et al., 2005, 
apud Cartwright, 2008). Comprovando, assim, a teoria de que pacientes borderline tiveram, em sua infância, pais que não proveram os cuidados necessários a seus filhos, deixando-os mais vulneráveis a possíveis riscos (Kernberg \& Michels, 2009; Beck, Freeman \& Davis, 2005; Lieb, Zanarini, Schmahl, Linehan \& Bohus, 2004; Scott, Levy \& Pincus, 2009; Helgeland \& Torgensen, 2004; Cartwright, 2008).

Esses achados provavelmente demonstram as dificuldades dos pais de pacientes borderline e o fato de muitos deles virem de famílias que já apresentam fatores de riscos importantes, como violência doméstica. Essas descobertas demonstram as grandes dificuldades desses pais em prover cuidados básicos à criança (Látolavá \& Prasko, 2010). Além disso, também fortalece a dificuldade de apego saudável, o que muitas vezes intensifica os sintomas do TPB, como dificuldades nos padrões de relacionamentos interpessoais (Scott, Levy \& Pincus, 2009)

A ligação com abuso sexual na infância é relacionada também com TEPT (Murray, 1993). Ainda que a relação de trauma com TPB não seja causal, é um dos fatores que têm um grande valor em sua etiologia (Helgeland \& Torgensen, 2004). Cantón-Cortés \& Cantón (2010) observaram que pessoas que sofreram abuso sexual na infância apresentaram níveis mais altos de presença de TEPT. $\mathrm{O}$ que se relaciona aos dados deste estudo, o qual demonstra altos níveis de sintomas de TEPT $(47,1 \%)$ nos pacientes borderline.

Observando os dados da amostra, também se ressalta correlação direta entre abuso emocional e abuso físico $(r s=, 434 ; p=0,025)$, assim é provável que pacientes que sofreram o abuso emocional também sofreram o físico. Além disso, sabe-se que os três tipos de abuso (sexual, físico e emocional) têm sido considerados como fatores de risco para o desenvolvimento do TPB (Axelrod, Morgan \& Southwick, 2005). Dados da pesquisa demonstram estar de acordo com esse achado, visto que $81 \%$ dos pacientes apresentaram algum tipo de abuso emocional. Quanto ao abuso físico, $66,6 \%$ relataram ter sofrido esse tipo de vivência traumática, fato que corrobora a teoria de que há relação desse tipo de abuso com sintomas de TPB e agressividade (Burnette \& Reppucci, 2009). Dessa forma, pessoas que sofreram abuso físico, quando crianças, podem confundir afeto com agressão, visto que entenderam as agressões sofridas como atenção.

A coocorrência entre negligência emocional (CTQ) e fator 3 excitabilidade do SPTSS, apesar de não demonstrar significância estatística $(r s=385 ; \mathrm{p}=0,042)$ nessa amostra, é importante ser discutida, tendo em vista que isso poderia estar relacionado com a teoria de que pacientes com TEPT podem ter sido vítimas de maus-tratos ou negligência na infância (Grassi-Oliveira \& Stein, 2008, apud Cantón-Cortés \& Cantón, 2010). Assim, poder-se-ia tentar explicar que, devido 
aos pacientes borderline perceberem essa negligência emocional como um tipo de trauma, isso acarretaria uma hipervigilância nas suas experiências de vida (ver Tabela 2).

Quanto ao TEPT, percebe-se uma presença significativa de comorbidade com TPB, pois $47,62 \%$ dos pacientes apresentaram, por meio do SPTSS, sintomas indicativos e intensos desse transtorno. Esse achado é condizente com o encontrado na literatura, no qual $75 \%$ dos pacientes com TPB apresentam TEPT como um diagnóstico comórbido (Zanarini et al., 1998; Zlotnick, Franklin \& Zimmerman, 2002; Gunderson, 2009).

Pode-se também demonstrar, ainda que de forma incipiente, que a teoria de que sintomas de TEPT, características do TPB e trauma estão relacionados entre si de maneira muito complexa, podendo a vivência traumática inclusive mudar a personalidade de uma pessoa (Axelrod, Morgan \& Southwick, 2005). Entretanto é necessário que se observe essa análise com muita cautela, pois as correlações nesse sentido demonstraram-se tímidas e o tamanho amostral desse estudo foi pequeno, o que denota a importância de novos estudos dentro dessa temática.

Quanto ao fator revivência $(\mathrm{M}=4,94 ; \mathrm{DP}=2,81)$, a literatura mostra que pode estar associado com experiências altamente emocionais. Os pacientes com TEPT desejam evitar futuros traumas, o que pode estar relacionado à alta incidência dos sintomas de evitação nos pacientes da amostra (Hagenaars, Minnen \& Hoogduin, 2009).

A forte ligação entre negligência física e abuso físico demonstra, de forma contundente nessa amostra, que as categorias de maus-tratos demonstraram-se presentes nos pacientes com TPB. Já a forte ligação de abuso emocional com abuso físico pode estar relacionada ao fato de ambos já poderem estar interligados, visto que uma criança pode entender um abuso físico como também sendo um abuso emocional e vice-versa. A correlação indireta entre a soma dos pontos da DIB-R e o fator de evitação do SPTSS (ver Tabela 2) pode ser explicada pelo usual padrão de impulsividade dos pacientes borderline bem como pelos comportamentos autodestrutivos destes. Os comportamentos impulsivos dessas pessoas tornam difícil evitar esses acontecimentos. Ao contrário dos pacientes com TEPT, que procuram evitar reviver ou lembrar o evento traumático, o paciente borderline tem os comportamentos inseridos em sua etiologia.

Ressaltamos, entretanto, que os achados necessitariam de uma amostra maior para que fossem mais bem apoiados teoricamente. Outro ponto a ser destacado se relaciona à necessidade de futuros estudos para que se analise se os sintomas de TEPT em pacientes borderline seriam, apenas, sintomas comórbidos ou um TEPT crônico. 


\section{Referências}

American Psychiatric Association (APA). (1987). Diagnostic and statistical manual of mental disorders. Porto Alegre: Artmed.

American Psychiatric Association (APA). (2002). Diagnostic and statistical manual of mental disorders. Porto Alegre: Artmed.

American Psychiatric Association (APA). (2013). Diagnostic and statistical manual of mental disorders. Washington: American Psychiatric Publishing.

Axelrod, S. R., Morgan, C. A. \& Southwick, S. M. (2005). Symptoms of posttraumatic stress disorder in veterans wars Operation Desert Sorm. American Journal of Psychiatry, 62, 270-275.

Ball, J. S. \& Links, P. S. (2009). Borderline personality disorder and childhood trauma: evidence for a causal relationship. Current Psychiatric Reports, 11, 638.

Beck, A., Freeman, T. \& Davis, D. (2005). Transtorno da personalidade borderline. In A. Beck, T. Freeman \& D. Davis, Terapia cognitiva dos transtornos da personalidade (pp. 167-188). Porto Alegre: Artmed.

Burnette, M. L. \& Reppucci, N. D. (2009). Child abuse and aggression in girls: the contribution of borderline personality disorder. Development and Psychopathology, 21, 309-317.

Cantón-Cortés, D. \& Cantón, J. (2010). Coping with child sexual abuse among college students and post-traumatic stress disorder: the role of continuity of abuse and relationship with the perpetrator. Child Abuse \& Neglect, 34, 496506.

Carlson, E. (2001). Psychometric study of a brief screen for PTSD: assessing the impact multiple traumatic events. Assessment, 8, 431-41.

Cartwright, D. (2008). Borderline personality disorder: what do we know? Diagnosis course, co-morbidity, and etiology. South African Journal of Psychology, 38, 429-446.

Esbec, E. \& Echeburúa, E. (2011). New criteria for personality disorders in DSM-V. Actas Españolas de Psiquiatría, 39(1), 1-11. 
Florenzano, R., Weil, K., Cruz, C., Acuña, J., Fullerton, C., Muñiz, C. ... Marambio, M. (2002). Personalidad Limítrofe, somatización, trauma y violencia infantil: un estudio empírico. Revista Chilena de Neuropsiquiatría, 40, 335-340.

Fruzzetti, A. E., Shenk, C. \& Hoffman, P. D. (2005). Family interaction and the development of borderline personality disorder: a transactional model. Development and Psychopathology, 17(4), 1007-1030.

Golier, J. A., Yehuda, R., Bierer, L. M., Mitropoulou, V., New, A. S., Schmeidler, J. ... Siever, L. J. (2003). The relationship of borderline personality disorder to posttraumatic stress disorder and traumatic events. American Journal of Psychiatry, 160(11), 2018-2024.

Grant, B. F., Chou, S. P., Goldstein, R. B., Huang, B., Stinson, F. S., Saha, T. D. ... Ruan, W. J. (2008). Prevalence, correlates, disability and comorbidity of DSM-IV borderline personality disorder: results from the Wave 2 National Epidemiologic Survey on Alcohol and Related Conditions. Journal of Clinic Psychiatry, 69(4), 533-545.

Grassi-Oliveira, R. \& Stein, L. M. \& Pezzi, J. C. (2006). Tradução e validação de conteúdo da versão em português do Childhood Trauma Questionnaire. Revista de Saúde Pública, 40(2), 249-255. Recuperado a partir de http://www. scielo.br/scielo.php?script=sci_arttext\&pid=S0034-89102006000200010\&ln $\mathrm{g}=$ en\&tlng=pt. $10.1590 /$ S0034-89102006000200010

Gunderson, J. G. (2009). Borderline personality disorder: ontogeny of a diagnosis. American Journal of Psychiatry, 166, 530-539.

Hagenaars, M. A., Minnen, A. V. \& Hoogduin, K. A. L. (2009). Reliving and disorganization in posttraumatic stress disorder and panic disorder memories. The Journal of Nervous and Mental Disease, 197, 627-630.

Helgeland, M. I. \& Torgersen, S. (2004). Developmental antecedents of borderline personality disorder. Comprehensive Psychiatry, 45(2), 138-147.

Hodges, S. (2003). Borderline personality disorder and posttraumatic disorder: time for integration? Journal of Counseling \& Development, 81(4), 409-417.

Johnson, A. B., Gentile, J. P. \& Correll, T. L. (2010). Acurately diagnosing and treating borderline personality disorder. Psychiatry Edgmont, 7(4), 21-30. 
Johnson, J. G., Smailes, E. M., Philip, M. \& Cohen, P. (2000). Associations between four types of childhood neglect and personality disorder symptoms during adolescence and early adulthood: findings of a community-based longitudinal study. ProQuest Medical Library, 14(2), 171-87.

Kaplan, H. I. \& Sadock, B.J. (1999). Tratado de psiquiatria. Porto Alegre: Artmed.

Kernberg, O. \& Michels, R. Borderline personality disorder. American Journal of Psychiatry, 166(5), 505-508.

Kernberg, O. F., Selzer, M. A., Koenigsberg, H. W., Carr, A, C. \& Appelbaum, A. H. (1991). Psicoterapia psicodinâmica de pacientes borderline. (R. C. S.Lopes, Trad.). Porto Alegre: Artmed. [Trabalho original publicado em 1975.]

Kristensen, C. H. (2005). Estresse pós-traumático: sintomatologia e funcionamento cognitivo (Tese de doutorado não publicada). Universidade Federal do Rio Grande do Sul, Curso de Pós-Graduação em Psicologia do Desenvolvimento, Porto Alegre.

Kudler, H. S. (1993). Borderline personality disorder and PTSD. American Journal of Psychiatry, 150(1), 19-27.

Látolavá, K. \& Prasko, J. (2010). Aggression in borderline personality disorder. Psychiatry Q., 81(3), 329-51.

Lieb, K., Zanarini, M. C., Schmahl, C., Linehan, M. M. \& Bohus, M. (2004). Borderline personality disorder. Lancet, 364, 453-461.

Linehan, M. (2010). Terapia cognitivo-comportamental para transtorno da personalidade borderline. (Ronaldo Cataldo Costa, Trad.). Porto Alegre: Artmed. [Trabalho original publicado em 1993.]

McLean, L. M. \& Gallop, R. (2003). Implications of childhood sexual abuse for adult borderline personality disorder and complex posttraumatic stress disorder. The American Journal of Psychiatry, 160, 369-371.

Murray, J. B. (1993). Relationship of Childhood sexual abuse to borderline personality disorder, posttraumatic stress disorder, and multiple personality disorder. The Journal of Psychology, 127(6), 657-676.

Oldham, J. M. (2009). Borderline personality disorder comes of age. American Journal Psychiatry, 166, 509-511. 
Pagano, M. E., Skodol, A. E., Stout R. L., Shea, M. T., Yen, S., Grilo, C.M. ... Gunderson, J. G. (2004). Stressful life events as predictors of functioning: findings from the Collaborative Longitudinal Personality Disorders Study. Acta Psychiatrica Scandinavica, 110(6), 421-429.

Pagura, J., Stein, M., Bolton, J., Cox, B., Grant, B., Sareen, J.(2010). Comorbidity of borderline personality disorder and posttraumatic stress disorder in the U.S. population. Journal of Psychiatric Research, 44(16), 1190-1198.

Sansone R. A. \& Sansone, L. A. (2009). Borderline personality and criminality. Psychiatric Edgmont, 6(10), 16-20.

Scott, L. N., Levy K. N. \& Pincus, A. L (2009). Adult attachment, personality traits, and borderline personality disorder features in young adults. Journal Personality Disorder, 23(3), 258-280.

Watson, D. C. \& Sinha, B. K. (1998). Comorbidity of DSM-IV Personality Disorders in a Nonclinical Sample. Journal of Clinical Psychology, 54(6), 773780 .

Widom, C. S., Czaja, S. J. \& Paris, J. (2009). A prospective investigation of borderline personality disorder in abused and neglected children followed up into adulthood. Journal of Personality Disorders, 23(5), 433-446.

Woodward, H. E., Taft, C. T., Gordon, R. A. \& Meis, L. A. (2009). Clinician Bias in the diagnosis of posttraumatic stress disorder and borderline personality disorder. Psychological Trauma: Theory, Research, Practice, and Policy, 1(4), 282-290.

Zanarini, M. C., Frankenburg, F. R., Dubo, E. D., Sickel, A. E., Trickha, A. et al. (1998). Axis I comorbidity of borderline personality disorder. American Journal of Psychiatry, 155(12), 1733-1739.

Zanarini, M. C., Frankenburg, F. R. \& Vujanovic, A. A. (2004). Axis II comorbidity of borderline personality disorder: description of 6-year course and prediction to time-to-remission. Acta Psychiatrica Scandinavica, 110, 416-420.

Zanarini, M. C., Gunderson, J. G., Frankenburg, F. R. \& Chauncey, D. C. (1989). The revised diagnostic interview for borderlines. Journal of Personality Disorders, 3, 10-18. 
Zlotnick, C., Franklin, C., Zimmerman, M. (2002). Is comorbidity of posttraumatic stress disorder and borderline personality disorder related to greater pathology and impairment? American Journal of Psychiatry, 159, 11.

Young, J. E., Klosko, J. S. \& Weishaar, M. E. (2008). Terapia do esquema: guia de técnicas cognitivo-comportamentais inovadoras. Porto Alegre: Artmed. 